\title{
Cognition of the relation between an event and a circumstance understood to explain the event $\dagger$
}

\author{
GORDON BEAR \\ University of Wisconsin, Madison, Wisconsin 53706
}

\begin{abstract}
A model is advanced for the process of explanation, according to which (a) an event to be explained is understood to be a value on a particular dimension of variation; (b) a circumstance believed to explain the event is understood to be a value on another dimension of variation; (c) the two dimensions are understood to be related such that the dimension whose value is to be explained is more likely to take that value when the dimension of circumstance takes its obtaining value than when the dimension of circumstance takes an alternative value. Evidence for the model is reported from a study in which Ss made two judgments about a human action-which of two statements of circumstance specifying alternative values for a certain dimension was the better explanation for the action, and which of the same two statements described the case in which the action, or for certain items the opposite action, was more likely to occur. For each of five different actions, the judgments were significantly related as required by the model.
\end{abstract}

Psychologists in recent years have devoted a great deal of their attention to human cognition. But as Singer (1971) has noted, there has been little psychological research directly concerned with a common cognitive process which appears to exert an important influence on human affairs-explanation. The most relevant work seems to be the social psychological research on "attribution," the seminal contributions to which are Heider's (1958), Jones and Davis's (1965), and Kelley's (1967). [Jones, Kanouse, Kelley, Nisbett, Valins, \& Weiner (1972) report more recent research.] But even though almost all of the psychologists concerned with attribution make reference to explanation, no one other than Kelley $(1967,1972,1973)$ seems to have analyzed the explanatory process in any detail. The present paper reports some work explicitly addressed to this problem. A model of certain features of the explanatory process is proposed here, and a study of explanations of human actions is reported which provides some evidence for the model's validity.

\section{TERMINOLOGY}

The process of interest here is that of explaining "why" a given state of affairs is the case, in the sense of showing how it came to be. This process should be distinguished from certain others which are also commonly called explanation, e.g., the explanation of "what" a particular symbol means. The term "explanation" also refers to the products of these processes, it should be noted; thus the product of the

TThe author wishes to thank David Mladenoff for help in the execution of the study reported here and Sharon Gurwitz for careful criticism of an earlier draft of this paper. Requests for reprints should be sent to Gordon Bear, Department of Psychology, University of Wisconsin, Madison, Wisconsin 53706. process of present interest, the answer to the "why" question, is also called an explanation.

The term explanandum, introduced by Hempel and Oppenheim (1948), is a convenient word, adjective or noun, for designating a state of affairs to be explained or a description of such a state of affairs. Thus one can speak concisely of an "explanandum event" described by an "explanandum sentence."

\section{A MODEL OF THE EXPLANATORY PROCESS}

The model advanced here derives from the presumption that a person understands a state of affairs to be one of a particular set of mutually exclusive alternatives, the others of which could have occurred but did not (cf. Garner, 1962; Olson, 1970; Strawson, 1952, pp. 3-7). A person may conceive the expansion of a metal coil, for example, as one possible event from a set whose other members are no change in the coil's size and contraction of the coil. The state of affairs which the person believes to have occurred and the alternatives to it which the person has in mind define a dimension of variation whose values are the mutually exclusive alternatives which he recognized, and thus, in this view, a person understands an event to be one particular value on a dimension of variation, and he will construe a sentence describing the event as a specification of a value on a dimension of variation.

The model is concerned only with three basic features of the explanatory process-the cognition of an event to be explained, the cognition of an explanatory circumstance, and the cognition of the relation between the two, the relation which makes the circumstance in fact explanatory for the cognizer. There is one postulate about each feature.

(1) An event to be explained is understood to be a 
value on a dimension of variation (the "explanandum dimension"). This postulate implies that a description of an event to be explained is construed as a specification of a value on a dimension of variation and that a person who seeks an explanation for an event is basically asking why a certain dimension of variation took a certain value rather than one of its alternative values, perhaps a specific alternative (cf. Hospers, 1960, p. 107). The person who observes that a metal coil has expanded, for example, may wonder why it has expanded rather than remaining the same size.

(2) A circumstance believed to explain an event is understood to be a value on a dimension of variation (an "explanatory dimension") other than the dimension on which the event is understood to constitute a value. This postulate implies that a statement of an explanatory circumstance is construed as a specification of a value on a dimension other than the explanandum dimension. Thus if the person wondering about the metal coil were told that it expanded because it was heated, he might take the heating to be an alternative to the maintenance of the coil at its original temperature and the cooling of the coil.

(3) An explanatory dimension is understood to be related to its explanandum dimension such that the explanandum dimension is more likely to take the value obtaining for it when the explanatory dimension takes its obtaining value than when the explanatory dimension takes any of its other values. This relation between the two dimensions is that which occurs in the causal schemata discussed in detail by Kelley $(1972,1973)$. The relation reflects a conception of causality which identifies a cause as that which is present when the effect occurs and makes a difference in the likelihood of the effect, in that the effect is less likely to occur when the cause is absent but other conditions remain constant. The person who understands that a metal coil expanded because it was heated, for example, would seem to be drawing on a schema relating the size of a piece of metal to its temperature. The schema indicates that metal expands (or is likely to do so) when heated but fails to expand (or is less likely to do so) when left alone or when cooled, assuming other conditions constant.

This model, it should be noted, is compatible with the description proposed by the philosopher of science Hempel (1965; Hempel \& Oppenheim, 1948) for the logical structure of an explanation. According to Hempel, an explanation for a state of affairs consists of a set of premises which logically imply a description of that state of affairs. The premises must include at least one statement of a particular circumstance and at least one general principle, Hempel says. The present model explicitly incorporates the statement of circumstance, of course, and the causal schema which the model posits corresponds to the general principle and supplies the cognition of an implication-like relation between the statement of circumstance and the explanandum statement.

\section{THE PRESENT STUDY}

The study reported here was intended to provide evidence on the cognition of the relation between an explanandum event and an explanatory circumstance. Ss were asked two questions about an event. The first was of the form "Which is the better explanation for the expansion of a metal coil--'It was heated' or 'It was not heated'?" The second was of the form "In which case is a metal coil more likely to expand-if it were heated or if it were not heated?" As the example illustrates, two circumstances representing different values on a dimension of variation were offered as potential answers to both questions. The third postulate of the present model states, in effect, that the schema used in explaining an event by reference to a circumstance is the same schema used in drawing an inference about its likelihood in that circumstance, and thus the answer to the question about explanation should be related in a certain way to the answer to the question about likelihood, if the model is correct. If the S holds a simple schema linking the dimension on which he understands the circumstance to vary and the dimension which he takes to be the explanandum, the circumstance which he regards as the better explanation for the event (probably the heating of the coil in the example) should also be the circumstance in which he judges the event to be more likely. If the S's schema is more complicated, though, and indicates the correlation between the two dimensions to hold only when certain other dimensions take certain values, and if he realizes that those values might not obtain for the event in question, then he should answer "It depends" to both questions. The response option "It depends" was accordingly added for both questions. On the other hand, if the $\mathrm{S}$ holds no relevant schema at all, he should judge the two circumstances to be equally good (actually equally poor) explanations and to make no difference for the likelihood of the event. The response options "Neither is a better explanation" and "It makes no difference" were accordingly added for the explanation and the likelihood questions, respectively. There were four choices for each question, then, and the prediction from the model is that a $\mathbf{S}$ will make corresponding choices in answering the two questions for a given event. Five events were tested; a complication introduced for two of them is described below.

The events were all human actions, a special concern of the research on attribution, and some basic remarks on the explanation of human action are required here. The cognition of human action appears to be based on a "rationality schema" stating a relationship among at least four dimensions: the actor's rationality, his decision about the action, his expectation about the outcome of the action, and his evaluation of the outcome. The schema can be paraphrased as the principle, "A rational actor chooses to cause what he desires and prevent what he fears, but refuses to cause 
what he fears or prevent what he desires; an irrational actor does the opposite." Similar formulations have been offered by a number of thinkers concerned with the cognition of behavior, including Heider (1958) and the philosophers Peters (1958), Hempel (1965), and Langford (1971). [Tolman (1932, 1959) and other psychologists, e.g., Irwin (1971), have proposed similar formulations as hypotheses about behavior itself.] In using the schema, a person ordinarily assumes the actor to be rational, it seems, and the person explains an action by reference to a state of affairs which the actor valued and believed he was causing or a state of affairs which the actor disvalued and believed he was preventing. The person's overt response, though, is likely to be elliptical, omitting the general principle, the assumption of rationality, and various details. ${ }^{1}$

\section{METHOD}

\section{Phase I}

In the first phase of the present study, explanations to serve as stimulus materials were collected for five human actions. The actions were arbitrarily chosen from among those relevant to the $S$ population of university students, and five explanandum sentences were composed, each attributing one of the actions to "a certain person," as shown in Table 1. Each of the five sentences was presented to a different $S$. The Ss were two males and three females who were arbitrarily selected from the persons studying in a library at the University of Wisconsin and were asked, "Would you have two minutes to help out with a psychology study? The study has to do with how people explain things. All we want is three explanations for a hypothetical situation." All five Ss agreed to help and were given a sheet of paper with one of the explanandum sentences typed in capital letters at the top and the instructions, "Please provide three possible explanations for this person's behavior. There is no need for you to give elaborate, creative explanations. Just give the kind of explanation you'd use in an ordinary conversation with a friend of yours." Each S did in fact write three sentences or sentence fragments. It was assumed that the instructions elicited the process of present interest, that the process operated on the explanandum sentence which the $S$ received, and that in the absence of special emphasis in the sentence, the $S$ took the verb of the sentence to specify the value of the dimension with which the sentence was concerned, thereby construing the explanandum event as the actor's performance of the given action, in contrast to his failure to do so (cf. Halliday, 1967, pp. 207-208; in Halliday's terms, the information focus is unmarked).

\section{Stimulus Materials}

The first explanations of the three provided by each S served as stimulus materials. All were statements of circumstance rather than general principles, as shown in Table 1. It might be noted parenthetically that, except for the response to the music action, the explanations are plausibly interpreted as products of the rationality schema and the assumption of the actor's rationality. For the travel, demonstration, and petition actions, the response asserts more or less explicitly that the actor valued a certain state of affairs, and in each case it seems a reasonable assumption that the $S$ presumed the actor to believe the action would cause this state of affairs. For the school action, the response states that the actor disvalued a certain state of affairs, and it seems a reasonable assumption that the $S$ presumed the actor to believe the action would prevent this state of affairs. For the music action, however, the response asserts that the action causes a state of affairs which is ordinarily disvalued (wasting time). The

\section{Table 1: Stimulus Materials}

Explanandum sentence, Phase I ... regularly listens to music Original explanation

Modified explanation

Alternative statement

it helps him to waste time.

It helps him to waste time.

It does not help him to waste time.

Explanandum sentence, Phase II ... regularly listens to music.

Inferendum sentence, Phase II ... would regularly listen to music?

Explanandum sentence, Phase I ... once signed a particular petition.

Original explanation

He feels strongly about something that personally affects him and wants something done about it.

Modified explanation

The petition has to do with something that personally affects him, he feels strongly about it, and he wants something done about it.

Alternative statement

The petition does not have to do with something that personally affects him, he does not feel strongly about it, and he does not want to see something done about it.

Explanandum sentence, Phase II ... once signed a particular petition.

Inferendum sentence, Phase II ... would sign a particular petition?

Explanandum sentence, Phase I ... once spent a summer traveling around the country.

Original explanation interest in seeing things outside of his realm of living.

Modified explanation He has an interest in seeing things outside of his realm of living.

Alternative statement He has no interest in seeing things outside of his realm of living.

Explanandum sentence, Phase II ... once spent a summer traveling around the country.

Inferendum sentence, Phase II ... would spend a summer traveling around the country?

Explanandum sentence, Phase I ... dropped out of school.

Original explanation probably sick of playing intellectual games.

Modified explanation He was sick of playing intellectual games.

Alternative statement He was not sick of playing intellectual games.

Explanandum sentence, Phase II ... stayed in school. Inferendum sentence, Phase II ... drop out of school?

Explanandum sentence, Phase I ... once participated in a stu-

Original explanation dent demonstration.

He was interested in the issue and felt he was "doing his part" by taking part in the demonstration.

Modified explanation Alternative statement He was interested in the issue. He was not interested in the issue.

Explanandum sentence, Phase II ... once participated in a student demonstration.

Inferendum sentence, Phase II ... not participate in a student demonstration?

Note-Each explanandum sentence began, " $A$ certain person . . ." and each inferendum sentence began, "In which case is it more likely that a certain person would...." 
present study was not intended to document the rationality schema and the assumption of the actor's rationality, though, and no attempt was made to test these interpretations of the explanations.

The explanations were modified, where necessary, to yield complete sentences with the following characteristics: (a) Each was about a male identified only by pronouns. (b) Each was in a tense appropriate to its explanandum sentence. (c) Each contained no qualifier such as "probably." In the absence of any special emphasis in the Ss' responses, it was assumed that the verb which each $S$ employed (implicitly if not explicitly) specified the value of the dimension of variation which he had in mind (the explanatory dimension), and a statement that this dimension took an alternative value was constructed by negating each verb of the modified explanation for four of the actions, as shown in Table 1. For the travel action, the alternative to "He has an interest in seeing things outside of his realm of living" was constructed not as "He does not have an interest ..." but as the semantically equivalent "He has no interest..." to prevent lengthening the sentence beyond one line of typescript in the booklets which presented the stimulus materials. For the demonstration action, the second verb of the explanation could not be sensibly negated when the first was (i.e., it is not sensible to say, "He was not interested in the issue and did not feel he was 'doing his part' by taking part in the demonstration"), so the second verb phrase was dropped.

\section{Phase II}

In the second phase of the study, 60 Ss were asked to perform an explanation task and an inference task using the explanations collected in the first phase of the study and the alternative statements generated from them. All instructions and materials for these tasks were presented in booklets, and the Ss indicated their judgments in writing.

Explanation Task. There were five pages for the explanation task, each cast in the following format:

\section{A CERTAIN PERSON ONCE SPENT A SUMMER} TRAVELING AROUND THE COUNTRY.

Which of the following two statements is the better explanation for this person's behavior? living.

He has no interest in seeing things outside of his realm of $\longrightarrow \mathrm{H}$ living.

Neither statement. (Neither statement is a better explanation than the other.)

- It depends. (Which statement is the better explanation depends on something.)

As the example shows, each page began with a sentence attributing a certain action to "a certain person." The sentence on four of the five pages was an explanandum sentence used in Phase $I$, as shown in Table 1 , but for reasons noted below, the sentence on the fifth page specified the action opposite to one used in an explanandum sentence in Phase I. Ss were instructed to choose "the better explanation for this person's behavior" and were offered two candidates for this choice. One candidate was the explanation for the behavior (or for its opposite) obtained in Phase I and modified as described above, and the other candidate was the alternative to this explanation generated as described above. Each page of the explanation task also offered two additional response choices, "Neither statement" and "It depends." The pages for the music action and the travel action each included paraphrases of the latter choices, given in parentheses as shown in the example. These actions were the first two which the Ss encountered in the explanation task, and the paraphrases were intended to clarify the meanings of the last two choices.
Inference Task. The five pages for the inference task were cast in a similar format:

In which case is it more likely that

A CERTAIN PERSON WOULD SPEND A SUMMER TRAVELING AROUND THE COUNTRY?

Case 1: He has an interest in seeing things outside of his realm of living.

Case 2: He has no interest in seeing things outside of his realm of living.

It makes no difference whether it's Case 1 or Case 2.

It depends. (In which case it's more likely, depends on something.)

As the example shows, each page asked in which case it is more likely that "a certain person" would perform a certain action. Four of the five pages specified an explanandum action used in Phase $I$, as shown in Table 1 , but for reasons noted below, the fifth page specified an action opposite to one used in Phase I. Ss were offered two candidates for the case in which the action was "more likely," and these were described by the same two statements offered as candidates for "the better explanation" of the same (or the opposite) action in the explanation task. Each page of the inference task also offered two additional choices, "It makes no difference whether it's Case 1 or Case 2," and "It depends." The pages for the music action and the travel action additionally included a paraphrase of the fourth choice, given in parentheses as shown in the example. These actions were the first two which the Ss encountered in the inference task, and the paraphrase was intended to clarify the meaning of the fourth choice.

Design and Predictions. For the travel action shown in the examples and for the music and petition actions, the same behavior was described in the explanation task and the inference task, and the explanandum action was thus the same as the "inferendum" action (the action to be inferred). For these item pairs, the model proposed here (in conjunction with the assumptions noted) makes the prediction indicated above-corresponding judgments on the two tasks. For the music and travel actions but not for the petition action, the order in which the two candidates for "the better explanation" were listed was reversed from that in which the two candidates for the case of greater likelihood were listed. Thus a $S$ conforming to the prediction and choosing between the two potential explanations and between the two cases would endorse different ordinal positions, and a simple position preference would not explain his data.

To provide a stricter test of the model, for the demonstration action and the school action, different behaviors were described in the explanation task and the inference task. As shown in Table 1, the demonstration action was "participating" for the explanation task and "not participating" for the inference task. The school action was "staying in" for the explanation task and "dropping out" for the inference task. In each case, one action was the alternative to the other. Thus the present model, in conjunction with the hypothesis that the rationality schema underlies the S's reasoning, requires that a $S$ who chooses one of the candidates for the "better explanation" should endorse the other candidate in the inference task and vice versa. The predictions for a S who chooses "Neither statement" or "No difference" or "It depends" are the same as for the other item pairs, however. For the demonstration action, the two statements were listed in the same orders for the two tasks, so that a $S$ conforming to the prediction and choosing between the potential explanations and between the two cases would endorse different ordinal positions. For the school action, the two statements were listed in opposite orders in the two tasks, so that the $S$ would endorse the same ordinal position.

Booklets. The five pages for the explanation task and the five pages for the inference task were assembled into a booklet with 
all pages for one task preceding the pages for the other task. Half of the Ss of each sex were given a booklet with the explanation task first; half were given a booklet with the inference task first. In all cases, the order of the actions involved in the judgments was music, travel, demonstration, petition, school, travel, music, demonstration, school, petition. At least three judgments thus intervened before a given action recurred.

Each booklet began with a cover page stating, "This is a study on how people reason." The page then requested the S's age and sex and continued, "We will be asking you 10 different questions. Please deal with these questions one at a time. Don't look back in the booklet, and don't look ahead. Treat each question as separate from the others. Answer each question by itself, without referring to any of the other questions. Thank you." The page concluded with a request to reread the instructions and a reminder not to look back in the booklet and not to look ahead in the booklet.

Subjects and Procedure. One male and one female E, working independently in one evening, selected Ss in a nonsystematic manner from among the persons studying in libraries of the University of Wisconsin who appeared to be native speakers of English. The Es showed potential Ss a card reading, "Could you spare five minutes to help out in a psychology study? The study has to do with how people reason. There's a questionnaire with just 10 questions." Nine persons refused the request on the card, but 60 (30 males and 30 females) accepted and completed a booklet. Ss reported ages ranging from 17 to 27 years, with a median value of 20.8 .

\section{RESULTS}

Four cases of a missing response and one case of a double response reduced the usable data to 295 pairs of judgments. Of these $295,63.4 \%$ were in accord with the predictions about the relation between the two kinds of judgments. The sex of the $S$, the order in which the $S$ made the judgments, and the interaction between sex and order did not significantly influence the probability of a S's confirming the predictions across the several actions, as shown in Table 2, and the data were accordingly collapsed across sex and order.

To determine whether the $63.4 \%$ of the judgment pairs which confirmed the predictions exceeded the proportion to be expected if the two judgments were unrelated, the 4 by 4 response matrices for the individual item pairs were examined. Table 3 presents the matrix for the pooled data. The frequencies with which judgment pairs were observed in the cells where the judgment pairs were predicted to fall was compared with the frequency expected for those cells on the null hypothesis that the two judgments were made independently, which is the hypothesis tested by the $\chi^{2}$ statistic. There was an excess of predicted judgment pairs amounting to about $19 \%$ of the total number for

Table 2

Mean Proportion of Confirming Judgment Pairs Per Subject

\begin{tabular}{llcc}
\hline & & \multicolumn{2}{c}{ Sex of Subject } \\
& & Male & Female \\
\hline \multirow{2}{*}{ Order of Judgments } & Explanation First & .590 & .620 \\
& Inference First & .720 & .597 \\
\hline
\end{tabular}

Note-N $=15 \mathrm{Ss}$ per cell. The datum analyzed was the proportion of the five judgment pairs (or the four pairs for $S$ s with missing data) which confirmed the predictions. MS error $=.081 . F$ for sex $<1, F$ for order $<1, F$ for sex by order $=1.09$.
Table 3

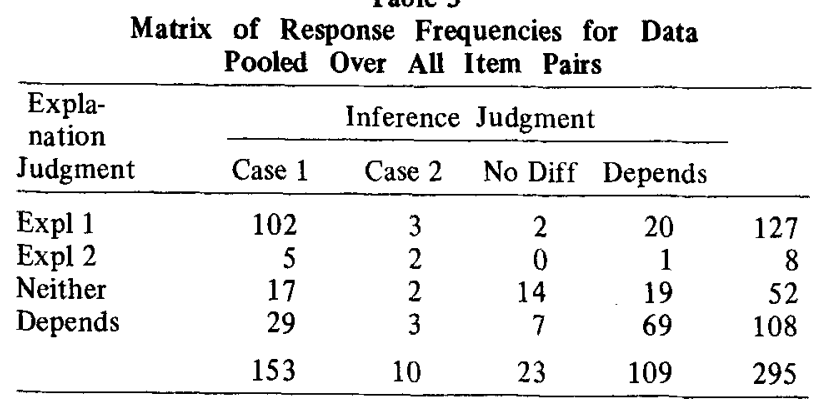

Note-Expl = explanation, neither $=$ neither statement, no diff $=$ it makes no difference, dopends $=$ it depends. The more popular explanation is in the first row, and the case which a $S$ choosing this explanation was predicted to select is in the first column. Judgment pairs are thus predicted to fall on the diagonal.

each item pair, as Table 4 shows, and the $\chi^{2}$ value was large enough to warrant rejection of the null hypothesis in each case. Thus for all five item pairs, including the two in which opposite actions were specified for the explanation task and the inference task, the S's two judgments proved to be significantly related in the way required by the model under test. The excess of confirming cases appeared in at least three of the four cells of the matrix where cases were predicted to fall for each item pair, it should be noted, indicating that, in general, no matter what response the $\mathrm{S}$ chose in making one type of judgment for a given action, he tended to confirm the predictions in making the other type of judgment for that action. This finding rules out the possibility that the excess of confirming cases occurred merely because the Ss were independently attracted to a particular response alternative in making their two judgments (or, for the special item pairs, independently attracted to a particular statement of circumstance in making one judgment and the opposite statement in making the other judgment).

The judgment pairs which departed from the predictions were divided into seven types for analysis. In order of frequency across the five item pairs, these were cases in which the first and second judgments were, respectively: (a) "It depends" and a statement of circumstance (35.2\% of the disconfirmations); (b) "It depends" and "Neither" or "No difference" (14.8\%); (c) a statement of circumstance and "It depends" $(13.9 \%)$; (d) "Neither" or "No difference" and a statement of circumstance (13.0\%); (e) "Neither" or "No difference" and "It depends (9.3\%); (f) a statement of circumstance and a statement of circumstance (7.4\%); (g) a statement of circumstance and "Neither" or "No difference" $(6.5 \%)$. In six of these seven categories, the bulk of the disconfirming cases proved to be due to just two of the item pairs, though not the same two for every category. Almost two-thirds of the cases of the most frequent type, $63.2 \%$ of them, came from the petition and demonstration pairs, which overall accounted for only $38.0 \%$ of the disconfirmations. Similarly, $62.5 \%$, $60.0 \%, 71.4 \%$, and $80.0 \%$ of the second through the fifth most common types, respectively, were due to just two other item pairs, school and music, which overall 
Table 4

Data on Individual Item Pairs

\begin{tabular}{|c|c|c|c|c|c|c|}
\hline & \multicolumn{5}{|c|}{ Item Pair } & \multirow[b]{2}{*}{ Total } \\
\hline & Music & Petition & Travel & School & Demonst & \\
\hline Percentage of Judgment Pairs Confirming Predictions & 57.6 & 69.0 & 79.3 & 50.0 & 61.7 & 63.39 \\
\hline Percentage of Confirming Pairs Expected by Chance & 39.2 & 51.5 & 58.8 & 30.3 & 41.5 & 44.12 \\
\hline Excess of Obtained Percentage Over Chance Percentage & 18.4 & 17.5 & 20.5 & 19.7 & 20.2 & 19.27 \\
\hline $\begin{array}{l}\chi^{2} \text { Testing Hypothesis that Chance Operated } \\
d f^{*} \text { for } \chi^{2} \\
p \text { value for } \chi^{2}\end{array}$ & $\begin{array}{l}24.76 \\
9 \\
.005\end{array}$ & $\begin{array}{l}16.62 \\
6 \\
.025\end{array}$ & $\begin{array}{l}28.78 \\
6 \\
.001\end{array}$ & $\begin{array}{l}13.52 \\
6 \\
.05\end{array}$ & $\begin{array}{l}20.95 \\
6 \\
.005\end{array}$ & \\
\hline
\end{tabular}

Note $-N=58,59$, or 60 judgment pairs for a given item pair.

*df $=9$ where all four response choices for each of the two judgments were selected at least once. df $=6$ where one choice for one of the judgments was not selected by any $S$.

accounted for only $50.9 \%$ of the disconfirmations, and $71.4 \%$ of the least common type was due to the school and travel pairs, which accounted for only $38.9 \%$ of the disconfirmations overall. Only for the sixth most common type of disconfirmation were the cases distributed across the five item pairs in close accord with the item pairs' overall contributions to the total number of disconfirmations.

\section{DISCUSSION}

The present data are generally encouraging to the model of explanation advanced above. For each of the five human actions tested, the S's judgment as to which of two statements of circumstance was the better explanation for the action was significantly related to his judgment as to which statement specified the case in which the action (or its opposite) was more likely to occur, and the relation between the two judgments was that required by the model, as shown by an excess of judgment pairs of the predicted types over the number to be expected if the judgments were not related.

Support for the model was far from complete, however, as $36.6 \%$ of the judgment pairs departed from the predictions. Unfortunately, the disconfirming cases of a given type were generally confined to only certain of the item pairs, and no one type of disconfirmation occurred frequently for each of the five pairs. The various disconfirmations thus seem to have resulted from various idiosyncrasies of the items, such as the semantically anomalous statements of circumstance "It helps ..." and "It does not help him to waste time" for the music pair. If the present procedure is used in future research, it would be desirable to employ a larger sample of item pairs and a larger sample of explanations for each pair, so that systematic departures from the model could be identified.

It would also seem desirable in future research to specify the alternative to the explanandum action and the inferendum action and thus eliminate ambiguity about just what the $S$ is to explain or infer. The $S$ might be asked to consider the fact that "A certain person spent a summer traveling around the country, instead of doing something else," for example. Similarly, each S in the first phase of the study who supplies an explanation to be tested in the second phase should be asked to indicate the alternative to the explanatory proposition which he has in mind, to eliminate the guesswork which was necessary in constructing the stimulus materials for the present experiment. Progress in understanding the explanatory process will require not only a superior model but a superior method to test it as well.

\section{REFERENCES}

Garner, W. R. Uncertainty and structure as psychological concepts. New York: Wiley, 1962.

Halliday, M. A. K. Notes on transitivity and theme in English: Part 2. Journal of Linguistics, 1967, 3, 199-244.

Heider, $\mathbf{F}$. The psychology of interpersonal relations. New York: Wiley, 1958.

Hempel, C. G. Aspects of scientific explanation. In C. G. Hempel, Aspects of scientific explanation and other essays in the philosophy of science. New York: Free Press, 1965. Pp. 331-496.

Hempel, C. G., \& Oppenheim, P. Studies in the logic of explanation. Philosophy of Science, 1948, 15, 135-175.

Hospers, J. What is explanation? In A. Flew (Ed.), Essays in conceptual analysis. Lond on: Macmillan, 1960. Pp.94-119.

Irwin. F. W. Intentional behavior and motivation: $A$ cognitive theory. New York: Lippincott, 1971.

Jones, E. E., \& Davis, K. E. From acts to dispositions: The attribution process in person perception. In $\mathrm{L}$. Berkowitz (Ed.), Advances in experimental social psychology. Vol. 2. New York: Academic Press, 1965. Pp. 219-266.

Jones, E. E., Kanouse, D. E., Kelley, H. H., Nisbett, R. E. Valins, S., \& Weiner, B. Attribution: Perceiving the causes of behavior. Morristown, N.J: General Learning Press, 1972.

Kelley, H. H. Attribution theory in social psychology. Nebraska Symposium on Motivation, 1967, 15, 192-238.

Kelley, H. H. Causal schemata and the attribution process. In $E$. E. Jones, D. E. Kanouse, H. H. Kelley, R. E. Nisbett, S. Valins, and B. Weiner, Attribution: Perceiving the causes of behavior. Morristown, N.J: General Learning Press, 1972. Pp. 151-174.

Kelley, H. H. The processes of causal attribution. American Psychologist, 1973, 28, 107-128.

Langford, G. Human action. Garden City, N.Y: Doubleday, 1971.

Olson, D. R. Language and thought: Aspects of a cognitive theory of semantics. Psychological Review, 1970, 77, 257-273.

Peters, R. S. The concept of motivation. London: Routledge \& Kegan Paul, 1958.

Singer, B. F. Toward a psychology of science. American Psychologist, 1971, 26, 1010-1015.

Strawson, P. F. Introduction to logical theory, London: Methuen, 1952.

Tolman, E. C. Purposive behavior in animals and men. New York: Century, 1932.

Tolman, E. C. Principles of purposive behavior. In S. Koch (Ed.) Psychology: A study of $a$ science. Vol. 2. New York: McGraw-Hill, 1959. Pp. 92-157.

\section{NOTE}

1. Bear, G., \& Martin, W. W. The assumption of rationality and the use of a means-end schema in the cognition of human action. In preparation.

(Received for publication August 1, 1973; revision received December 20,1973 .) 